\title{
Electrothermally induced fluid flow on microelectrodes
}

\author{
Nicolas G. Green ${ }^{\mathrm{a}}$, Antonio Ramos ${ }^{\mathrm{a}, *}$, Antonio González ${ }^{\mathrm{a}}$, \\ Antonio Castellanos ${ }^{\mathrm{a}}$, Hywel Morgan ${ }^{\mathrm{b}}$ \\ a Dpto. Electronica y Electromagnetismo, Facultad de Fisica, Universidad de Sevilla, \\ Avenida Reina Mercedes s/n, 41012 Sevilla, Spain \\ ${ }^{\mathrm{b}}$ Bioelectronics Research Centre, Dept. Electronics and Electrical Engineering, University of Glasgow, \\ Rankine Building, Oakfield Avenue, Glasgow G12 8LT, Scotland, UK
}

\begin{abstract}
Planar microelectrodes, used for the electrokinetic manipulation of particles, generate high strength AC electric fields, resulting not only in forces on the particles but also on the suspending fluid. Observations of electrolytes on microelectrode structures at applied signal frequencies of the order of $1 \mathrm{MHz}$ have shown the importance of the illumination in generating fluid flow. In this paper, these experiments are analysed in terms of the theory of electrothermally induced fluid flow. Numerical calculations are made of the electric field, temperature field and fluid flow, arising both from Joule heating and from light heating. The results verify that Joule heating is not important under the experimental conditions. The temperature gradient generated by the light that is required in order to match the experimental fluid velocities is determined. (C) 2001 Elsevier Science B.V. All rights reserved.
\end{abstract}

Keywords: Electrohydrodynamics; Microelectrodes; Electroconvection; Thermally induced flow electrolytes

\section{Introduction}

Microelectrode structures are commonly used in AC Electrokinetics to generate the high strength $\mathrm{AC}$ electric fields required to move colloidal particles suspended in liquid [1]. A wide range of sizes of particles have been dielectrophoretically

\footnotetext{
*Corresponding author. Tel.: + 34-954-550-960; fax: + 34-954-239-434.

E-mail address: ramos@cica.es (A. Ramos).
} 
manipulated in this manner, from cells $(\sim 10 \mu \mathrm{m})$ and bacteria down to viruses $(\sim 100 \mathrm{~nm})$ and protein molecules [2,3]. The fields also result in flow in the suspending fluid through a number of mechanisms. One mechanism, important at frequencies below $\sim 100 \mathrm{kHz}$, is $\mathrm{AC}$ electroosmosis, which arises from electrical body forces in the diffuse double layer [4,5].

This paper discusses observations of fluid flow on microelectrode structures at higher frequencies, where charges do not have sufficient time to accumulate in the diffuse double layer. Other authors have reported movement of fluid at these frequencies [6,7] and attributed this to electrothermal pumping, with the temperature gradients arising from Joule heating. We have identified a second mechanism of heating in the system: the light used to observe the electrodes [6] and in our experiments this seems to be a stronger effect than Joule heating.

As described in more detail in related publications [8], for observation of the fluid flow, we used a simple electrode design consisting of two plates, just under $2 \mathrm{~mm}$ long and $0.5 \mathrm{~mm}$ wide, with parallel edges separated by $25 \mu \mathrm{m}$ and fabricated on planar glass substrates. Fluorescent latex spheres of a variety of sizes were used as tracer particles to observe the movement of the fluid. These particles were suspended in aqueous solutions of potassium chloride and the electrodes were illuminated and observed using a fluorescent microscope. The particles were observed both in the horizontal plane and in a vertical plane using a second microscope pointing along the electrodes [8]. For an applied potential of $10 \mathrm{~V}$ and $1 \mathrm{MHz}$, a typical value of the fluid velocity was $80 \mu \mathrm{m} / \mathrm{s}^{-1}$. This velocity was significantly reduced if either the intensity of the illumination or the applied voltage was reduced. Using an image intensified camera, we noted that the fluid flow ceased altogether for very low levels of illumination. A clear indication that this fluid flow was electrothermal in origin was that it was frequency dependent and changed direction at the characteristic frequency given by the theory [8,9].

In order to discount direct heating of the water by infrared light, an IR filter was placed in front of the mercury lamp. In addition, the experiments were conducted using epi-fluorescence microscopy with band-pass filters which only allowed light of a given wavelength range to reach the sample. Maximum fluid flow was observed for green light, centre wavelength $500 \mathrm{~nm}$. Using a disk thermocouple (diameter $1 \mathrm{~mm}$, thickness approximately $100 \mu \mathrm{m}$ ) placed at the focal point of the illumination, a temperature rise of $15^{\circ} \mathrm{C}$ was recorded, which correlates with values determined by the numerical calculations presented.

In this paper, the approximations to the equations governing electrothermally induced fluid flow for the experimental microelectrodes are described. A commercially available partial differential equation solver based on the Finite Element Method, FlexPDE [10], is used to numerically calculate the electric field, thermal field and the fluid velocity arising from both Joule heating and the illumination source. The magnitude and importance of Joule heating as a source of fluid flow on microelectrodes is examined. The results are then compared with experiments and some conclusions are drawn about the temperature field required for the measured velocities. 


\section{Theory}

Electrothermal fluid flow arises from the action of the electric field on inhomogeneities in the medium induced by temperature gradients. The velocity of the fluid, $\mathbf{u}$, is governed by Navier-Stokes' equation in the low Reynolds number approximation:

$$
0=-\nabla p+\eta \nabla^{2} \mathbf{u}+\mathbf{f}_{e}+\Delta \rho_{m} \mathbf{g}
$$

together with the mass conservation equation for an incompressible fluid:

$$
\nabla \cdot \mathbf{u}=0
$$

where $p$ is the pressure, $\eta$ is the viscosity of the fluid, $\Delta \rho_{m} \mathbf{g}$ is the buoyancy force density and $\mathbf{f}_{e}$ is the electric force density. The low Reynolds number approximation is a valid assumption for microelectrode structures, since for typical experimental values (velocity $u \sim 50 \mu \mathrm{m} \mathrm{s}^{-1}$, distance $l \sim 10 \mu \mathrm{m}$, viscosity $\eta \sim 10^{-3} \mathrm{~kg} \mathrm{~m}^{-1} \mathrm{~s}^{-1}$, mass density $\rho_{m} \sim 10^{3} \mathrm{~kg} \mathrm{~m}^{-3}$ ), the Reynolds number, $\rho_{m} u l / \eta \sim 5 \times 10^{-4}$. The inhomogeneities in the viscosity caused by the temperature field are considered to be small, so we have not included a term for the gradient of viscosity in the NavierStokes equation.

\subsection{The electrical force density}

The electrical force density is given by [11]:

$$
\mathbf{f}_{e}=\rho_{q} \mathbf{E}-\frac{1}{2} E^{2} \nabla \varepsilon+\frac{1}{2} \nabla\left(\rho_{m}\left(\frac{\partial \varepsilon}{\partial \rho_{m}}\right)_{T} E^{2}\right),
$$

where $\rho_{q}$ is the charge density, $\varepsilon$ is the permittivity, $T$ is the temperature and $E$ is the magnitude of the electric field $\mathbf{E}$. The first and second terms are the Coulomb and dielectric forces, respectively. The last term is the electrostriction, which can be combined with the pressure term in Eq. (1) and omitted from the calculation.

The charge density and the electric field are described by Gauss's law

$$
\rho_{q}=\nabla \cdot(\varepsilon \mathbf{E})
$$

the charge conservation equation

$$
\frac{\partial \rho_{q}}{\partial t}+\nabla \cdot\left(\rho_{q} \mathbf{u}\right)+\nabla \cdot(\sigma \mathbf{E})=0
$$

and

$$
\nabla \times \mathbf{E}=0,
$$

where $\sigma$ is the conductivity of the fluid. The ratio of magnitudes of the convection current, $\rho_{q} \mathbf{u}$, to the conduction current, $\sigma \mathbf{E}$, is given by the Electrical Reynolds number $u \varepsilon / \sigma l$ [12]. For typical values, this is $3 \times 10^{-7}$ so that the second term in Eq. (5) can be neglected.

The relative changes in the permittivity and conductivity are small for typical temperature increments [9] and we can perform a perturbative expansion. In the 
lowest order, Eqs. (4) and (5) reduce to $\rho_{q}=0$ and $\nabla \cdot \mathbf{E}_{0}=0$, so that the electric potential obeys Laplace's equation. The first order charge density can then be obtained in terms of $\mathbf{E}_{0}$ as follows. For an AC field of frequency $\omega$, Eqs. (4) and (5) can be combined to give the following expression, which relates the charge density phasor to the electric field phasor:

$$
\rho_{q}(\sigma+\mathrm{i} \omega \varepsilon)=\sigma \nabla \varepsilon \cdot \mathbf{E}_{0}-\varepsilon \nabla \sigma \cdot \mathbf{E}_{0},
$$

where $\mathrm{i}=\sqrt{-1}$. Here, we have assumed that the changes in the permittivity and conductivity do not depend on time. Since the gradients in $\sigma$ and $\varepsilon$ are due to the temperature gradients, we can write Eq. (7) as

$$
\rho_{q}=\frac{\sigma \varepsilon(\alpha-\beta)}{\sigma+\mathrm{i} \omega \varepsilon} \nabla T \cdot \mathbf{E}_{0}
$$

where

$$
\alpha=\frac{1}{\varepsilon}\left(\frac{\partial \varepsilon}{\partial T}\right) \text { and } \beta=\frac{1}{\sigma}\left(\frac{\partial \sigma}{\partial T}\right) .
$$

For an aqueous $\mathrm{KCl}$ solution, $\alpha \approx-0.4 \% \mathrm{~K}^{-1}$ and $\beta \approx 2 \% \mathrm{~K}^{-1}$ [13]. To first order, the time averaged electrical force density is therefore [9]

$$
\left\langle\mathbf{f}_{e}\right\rangle=\frac{1}{2} \operatorname{Re}\left(\frac{\sigma \varepsilon(\alpha-\beta)}{\sigma+\mathrm{i} \omega \varepsilon}\left(\nabla T \cdot \mathbf{E}_{0}\right) \mathbf{E}_{0}^{*}-\frac{1}{2} \varepsilon \alpha\left|\mathbf{E}_{0}\right|^{2} \nabla T\right),
$$

where * indicates complex conjugate and $\operatorname{Re}(. .$.$) the real part of (...). For an AC$ signal of frequency $\omega$, the electric field and the charge density oscillate with the same frequency. The force contains products of these magnitudes and therefore contains both a steady term (given by Eq. (9)) and a term that oscillates at $2 \omega$. The resulting distributions of $\mathbf{u}$ and $p$ also have both steady and oscillating terms but, at sufficiently high frequencies, the oscillatory are much smaller than the steady. Therefore, we only consider the time averaged force in the Navier-Stokes' equation.

As discussed in Ref. [9], the force density is frequency dependent and has two distinct limits: at low frequencies the Coulomb force (the first term on the right hand side of (9)) dominates and at high frequencies the dielectric force dominates. Typically, these two forces act in different directions and over a certain range of frequencies they compete, resulting in a changing flow pattern [9].

\subsection{Sources of heat}

In general, the electric, temperature and velocity fields are coupled. In order to solve the temperature field, the energy balance equation must be used $[14,15]$

$$
\rho_{m} c_{\mathrm{p}} \frac{\partial T}{\partial t}+\rho_{m} c_{p} \mathbf{u} \cdot \nabla T=k \nabla^{2} T+\sigma E^{2},
$$

where $c_{\mathrm{p}}$ is the specific heat at constant pressure, $k$ is the thermal conductivity and $\sigma E^{2}$ is the Joule heating term. Here, we have neglected the viscous dissipation term $[14,15]$, which is of the order of $10^{-10}$ times smaller than the Joule heating term. For sufficiently high frequencies, we can simplify this equation to the steady state [9]. 
Also, the ratio of convection of heat to heat diffusion is very small: $\rho_{m} c_{\mathrm{p}} u l / k \sim$ $3 \times 10^{-3}$ and we can neglect the second term on the left-hand side. The temperature is therefore governed by the diffusion equation with a source term given by the time average of the Joule heating: $\left\langle\sigma \mathbf{E}^{2}\right\rangle$.

\subsection{Summary of equations}

As a result of this set of approximations, the electrical, thermal and mechanical problems are decoupled and we solve the complete problem as follows. First we solve the electrical problem: Laplace's equation for the potential in a homogeneous medium.

$$
\nabla^{2} \phi=0, \quad \mathbf{E}=-\nabla \phi .
$$

Second we calculate the temperature field using the solution for the electric field,

$$
k \nabla^{2} T+\left\langle\sigma E^{2}\right\rangle=0 .
$$

Finally, we use these two solutions to compute the electrical force and solve the Navier-Stokes' equation for the fluid velocity and pressure. The force due to buoyancy is negligibly small compared to the electrical force [9] and the NavierStokes equation is

$$
-\nabla p+\eta \nabla^{2} \mathbf{u}+\left\langle\mathbf{f}_{e}\right\rangle=0
$$

\section{Numerical model}

For the numerical solution of the equations, we used a commercially available Finite Element partial differential equation solver, FlexPDE [10,16]. In general, a 2D problem space is divided into triangular elements and the variables are approximated by second or third order polynomials in each element (we have used third order polynomials). The program employs an adaptive mesh refinement technique to improve the accuracy of the solution. In order to solve the Navier-Stokes equation, we used the penalty method given in Ref. [16].

The experimental system to be modelled consists of thin planar electrodes fabricated on a microscope slide and a covering layer of fluid enclosed by a chamber constructed from glass cover slips. Several simplifications can be made to this system.

The electrodes were assumed to be infinitely long, so that the problem space was restricted to the $2 \mathrm{D}$ cross-section. This is a suitable approximation since the electrodes were just under $2 \mathrm{~mm}$ in length and $500 \mu \mathrm{m}$ in width. The electrodes are very thin $(\sim 100 \mathrm{~nm})$ in comparison to their width and were assumed to be infinitely thin. The system is symmetrical about the centre of the gap between the electrodes and only half of the problem is required in the problem space. The resulting $2 \mathrm{D}$ problem space is shown in Fig. 1. The unknowns of the problem are the electrical potential $\phi$, the temperature $T$, and the horizontal and vertical velocities $u$ and $v$, respectively. 


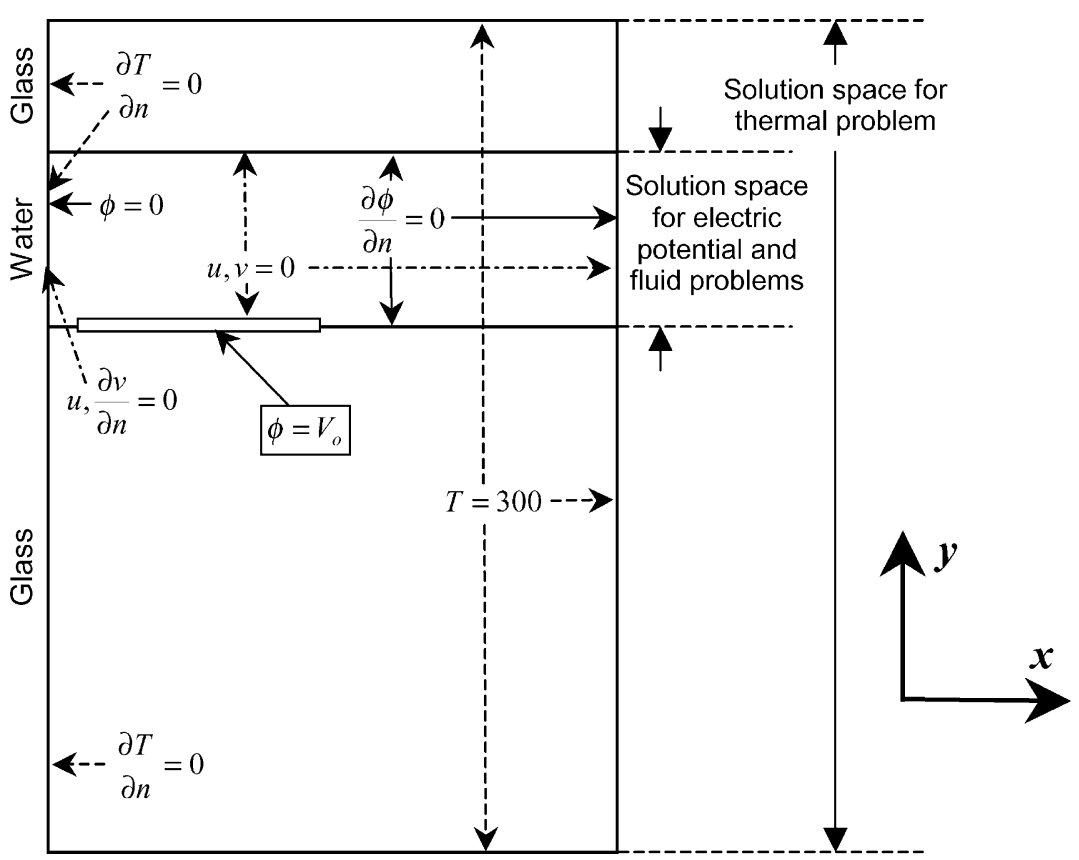

Fig. 1. Schematic diagram of the problem space and the general boundary conditions for the electrical, thermal and fluid dynamic problems.

\subsection{The electrical problem}

The large difference between the experimental conductivities and permittivities of the water solution $\left(\sigma=2.1 \times 10^{-3} \mathrm{~S} \mathrm{~m}^{-1}, \varepsilon=80 \varepsilon_{0}\right)$ and the glass $\left(\sigma=0, \varepsilon=3 \varepsilon_{0}\right)$, allows us to consider only the fluid in the electrical problem. Using the requirement that the total normal current across the interface must be continuous:

$$
\left[(\sigma+\mathrm{i} \omega \varepsilon) \frac{\partial \phi}{\partial n}\right]_{\text {water }}=\left[(\sigma+\mathrm{i} \omega \varepsilon) \frac{\partial \phi}{\partial n}\right]_{\text {glass }}
$$

it can be seen that the Neumann condition $\partial \phi / \partial n=0$, where $n$ is the normal to the boundary, holds in the fluid at the interfaces between the water and the glass. The electrode was assigned to be at a fixed potential $V_{0}$ and the boundary at the left was assigned to be a plane of odd symmetry $(\phi=0)$. The remaining boundary is placed far from the electrode and is assigned the condition $\partial \phi / \partial n=0$. A detailed discussion of the validity of the boundary condition can be found in Appendix A.

\subsection{The thermal problem}

The thermal conductivities of the glass and the water are 1.0 and $0.6 \mathrm{Jm}^{-1} \mathrm{~s}^{-1} \mathrm{~K}^{-1}$, respectively. The outer surfaces of the system were assumed to be at a constant temperature (room temperature $300 \mathrm{~K}$ ). The left-hand boundary was assigned to be a 
plane of even symmetry: $\partial T / \partial n=0$ since the magnitude of the electric field has this symmetry with respect to this boundary.

We have performed two classes of computations: one where only the Joule heating is present, and a second one where the illumination is taken into account as source of heat, with or without Joule heating.

In the case of Joule heating, there are a variety of possible electrode boundary conditions for the thermal problem. Two limit cases are considered. First, assuming that the electrodes can conduct heat in the $z$-direction (perpendicular to the paper in Fig. 1) much better than the fluid and the glass, then they are at the external (room) temperature. Second, we assume that, since the electrodes are very thin, they have a thermal resistance greater than the fluid in both the $x$-direction and the $z$-direction and a negligible resistance in the $y$-direction. As a result, the electrodes can be assumed to be transparent for the flux of heat and are removed from the model for the calculation of the temperature field. The second case is closer to the experiments.

To include the light as a source of heat, we have assumed that the electrodes are at a higher temperature than the room temperature. There are a number of mechanisms through which the light could transfer energy to the system. There could be absorption in the glass, the water, the electrodes, or even the particles. However, while the temperature field could be created by a combination of these effects, previous simple calculations indicate that there is a gradient of temperature in the fluid directed towards the illuminated electrodes [8].

\subsection{The fluid-mechanical problem}

The viscosity of the fluid is $0.001 \mathrm{~kg} \mathrm{~m}^{-1} \mathrm{~s}^{-1}$ at $20^{\circ} \mathrm{C}$. The boundary conditions for the mechanical problem are: (i) the no-slip boundary condition at the bottom, top and right hand edges; and (ii) that both the horizontal velocity $u$ and the normal derivative of the vertical velocity $\partial v / \partial n$ are zero at the symmetry plane.

\section{Results and discussion}

The calculated electrical potential is shown in Fig. 2a and the electric field magnitude is shown in Fig. 2b. The electric field strength is a maximum at the electrode edge and decreases rapidly with increasing distance as expected.

The following calculations of the fluid velocity and comparisons with experimental velocities are for the low frequency limit. Here, by low frequency limit we mean frequencies at which the Coulomb force (as described by Eq. (9)) is dominant. The characteristic frequency at which the behaviour changes from being Coulomb force dominated to dielectric force dominated is given by [9]:

$$
\omega \approx \frac{\sigma}{\varepsilon}\left|2 \frac{1}{\sigma}\left(\frac{\partial \sigma}{\partial T}\right) / \frac{1}{\varepsilon}\left(\frac{\partial \varepsilon}{\partial T}\right)\right|^{1 / 2} .
$$

For our experimental conditions this frequency is $f=\omega / 2 \pi=1.52 \mathrm{MHz}$ [8]. 

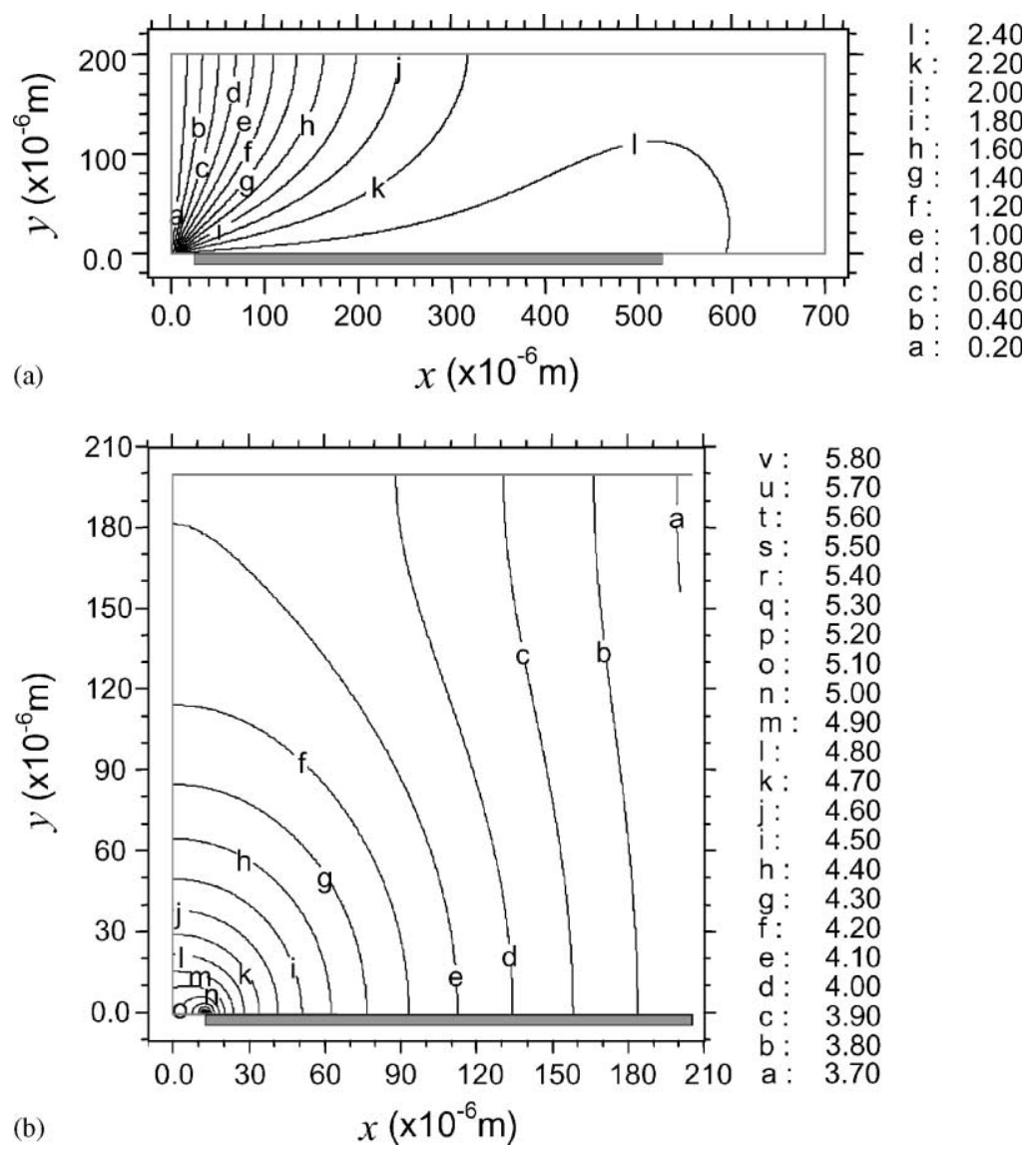

Fig. 2. (a) The equipotential lines for the solved electrical potential plotted as a contour plot with the values of the contours given on the right in volts. The electrode lies between $x=12.5 \mu \mathrm{m}$ and $521.5 \mu \mathrm{m}$ as shown in the figure. The voltage applied to the electrode shown was $2.5 \mathrm{~V}$. (b) The electric field magnitude calculated from the potential for the $200 \mu \mathrm{m}$ by $200 \mu \mathrm{m}$ region at the gap between the electrodes. The magnitude is plotted as a contour plot with the value of each contour shown by the scale on the right. The scale is $\log _{10}$ of the field magnitude so that along contour $\mathbf{f}$ the magnitude of the electric field is $10^{4.2} \mathrm{~V} \mathrm{~m}^{-1}$. The electrode edge is at $x=12.5 \mu \mathrm{m}$.

\subsection{Joule heating}

Fig. 3 shows the numerically calculated thermal and velocity fields for Case A, electrode at room temperature. Fig. 4 shows the same fields for Case B, electrode not present in calculation of temperature. In both cases, the highest temperature is achieved at a point above the centre of the electrode, with a higher temperature for Case B. In spite of this, the typical gradient of temperature is greater for Case A since the electrode is at room temperature. This produces a larger body force on the fluid and therefore, larger velocities for Case A. The velocity graphs (Figs. 3b and 4b) show circulating patterns, with the fluid moving down in the centre of the gap and 

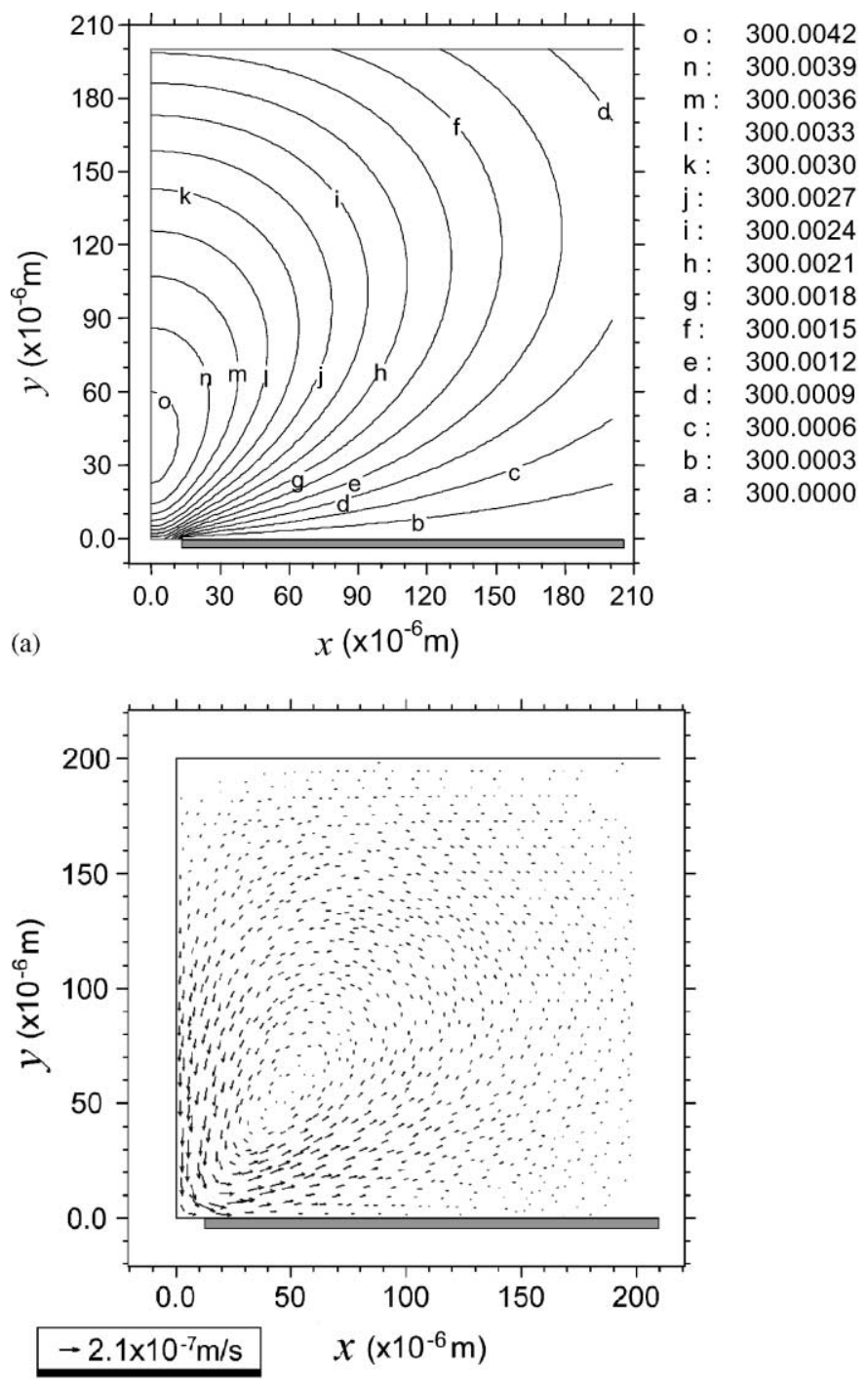

(b)

Fig. 3. (a) The thermal field in the fluid arising from Joule heating in the $200 \mu \mathrm{m}$ by $200 \mu \mathrm{m}$ region starting from the centre of the gap between the electrodes. This figure is for low electrode thermal resistance (Case A) and the data is plotted as isothermal lines with the scale shown on the right (in Kelvin). The edge is at $x=12.5 \mu \mathrm{m}$ and the applied voltage had an amplitude of $5 \mathrm{~V}( \pm 2.5 \mathrm{~V}$ on each electrode). (b) A vector plot of the fluid velocity profile arising as a result of the thermal field in Fig. 3a (Case A). The electrode is marked in the figure and the length of the arrow in the margin gives the scale for the velocity (linear). The amplitude of the voltage was $5 \mathrm{~V}( \pm 2.5 \mathrm{~V}$ on each electrode $)$. 

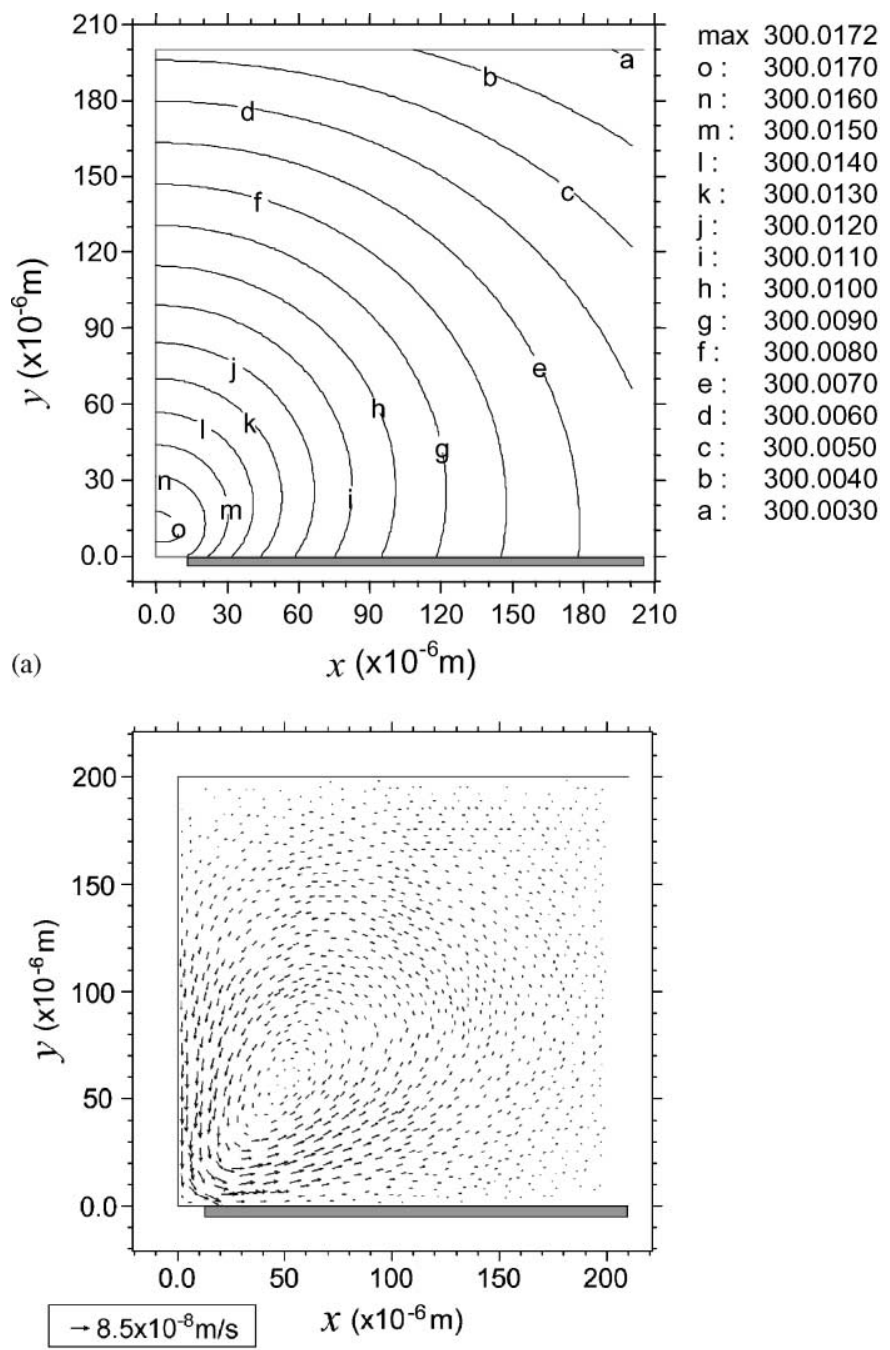

(b)

Fig. 4. (a) The thermal field in the fluid arising from Joule heating for the electrode with high thermal resistance (Case B). The plot shows isothermal lines (in Kelvin, with scale on right) in the $200 \mu \mathrm{m} \times 200 \mu \mathrm{m}$ region starting from the centre of the gap between the electrodes. The amplitude of the voltage was $5 \mathrm{~V}$ ( $\pm 2.5 \mathrm{~V}$ on each electrode). (b) A vector plot of the fluid velocity profile arising as a result of the thermal field in Fig. 4a (Case B). The electrode is marked in the figure and the length of the arrow in the margin gives the scale for the velocity (linear). The voltage amplitude was $5 \mathrm{~V}( \pm 2.5 \mathrm{~V}$ on each electrode).

out over the electrode surface. These velocity fields were calculated for the low frequency limit.

The theory predicts that the velocity will be proportional to $\sigma V^{4}$, since the heat generation (and $\Delta T$ ) is proportional to $\sigma V^{2}$ and the electrical stress is proportional to $\Delta T V^{2}$. Using the scaling factors $\sigma V^{2}$ for the thermal field and $\sigma V^{4}$ for the 
velocities, the velocity for any conductivity and voltage can be calculated from one numerical solution. For an applied signal of amplitude $10 \mathrm{~V}$ and a conductivity of $2.1 \times 10^{-3} \mathrm{~S} \mathrm{~m}^{-1}$ and using the analytical solution given in [9], a typical velocity at $40 \mu \mathrm{m}$ above the centre of the gap is $7 \mu \mathrm{ms}^{-1}$ at low frequencies. Numerical calculations give values of $3.2 \mu \mathrm{m} \mathrm{s}^{-1}$ for Case A and $1.3 \mu \mathrm{m} \mathrm{s}^{-1}$ for Case B.

These calculated velocities are much smaller than those observed at high light intensities for the same experimental conditions $\left(\sim 80 \mu \mathrm{m} \mathrm{s}^{-1}\right)$ [8]. In addition, the direction of the circulating motion is opposite to the experimental results in the same limit, verifying that Joule heating is not responsible for the observed fluid motion.

\subsection{Light heating}

The effect of light heating was modelled by defining the illuminated electrodes to be at a higher temperature than the room temperature. As discussed in Ref. [8], the direction of the force and the resulting fluid flow based on this assumption agrees with experimental observations.

Fig. 5 shows the numerically calculated thermal field in the system, demonstrating that the gradient of temperature is almost vertical in the region over the electrodes. Fig. 6 shows the numerically calculated velocity for a potential of $5 \mathrm{~V}$ for this thermal field. Again, there is a circulating pattern but in the opposite direction to the Joule heating cases. Fig. 7 shows the streamlines calculated from the velocity field. Comparison of this figure and the photograph (Fig. 2) in Ref. [8] demonstrates that the calculated pattern of the flow is in good agreement with the experimental pattern.

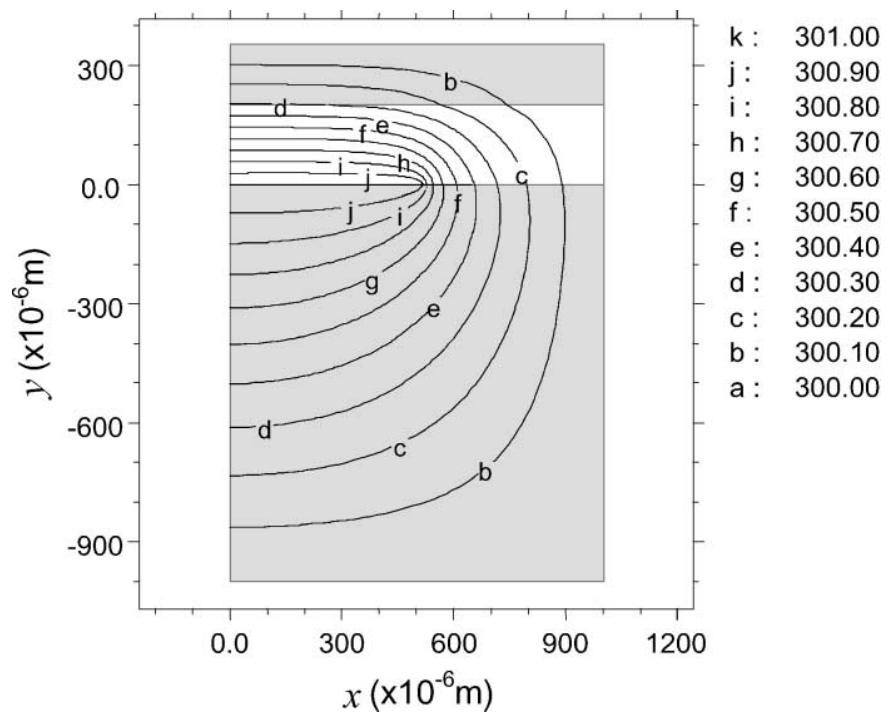

Fig. 5. The thermal field in the entire problem space for an electrode at a fixed temperature. In this figure the electrode is placed between $(12.5,0)$ and $(512.5,0)$. The plot is of the isothermal lines with the scale given on the right (in Kelvin). 


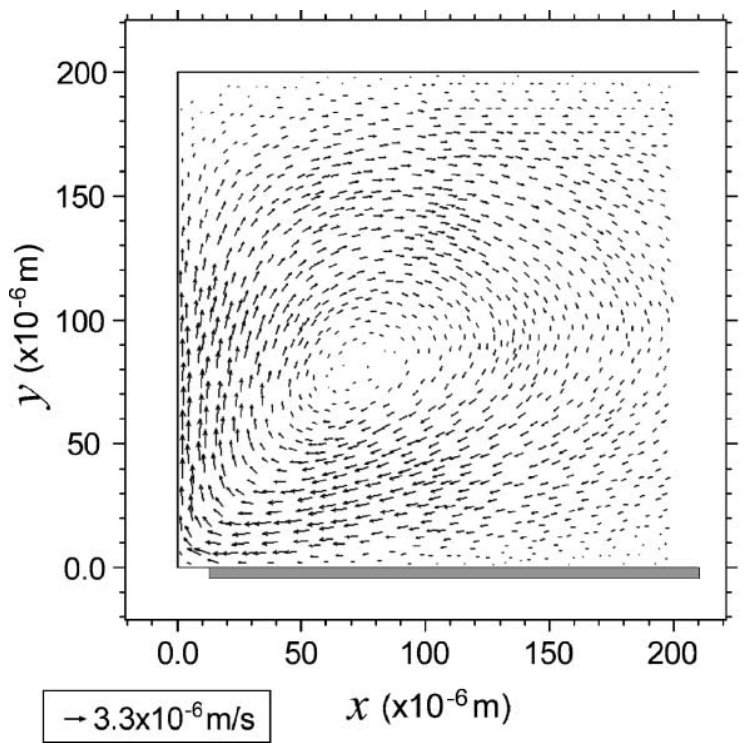

Fig. 6. A vector plot of the fluid velocity profile arising as a result of the thermal field in Fig. 5 for the heated electrode. The electrode is marked in the figure and the length of the arrow in the margin gives the scale for the velocity (linear). The amplitude of the voltage was $5 \mathrm{~V}( \pm 2.5 \mathrm{~V}$ on each electrode).

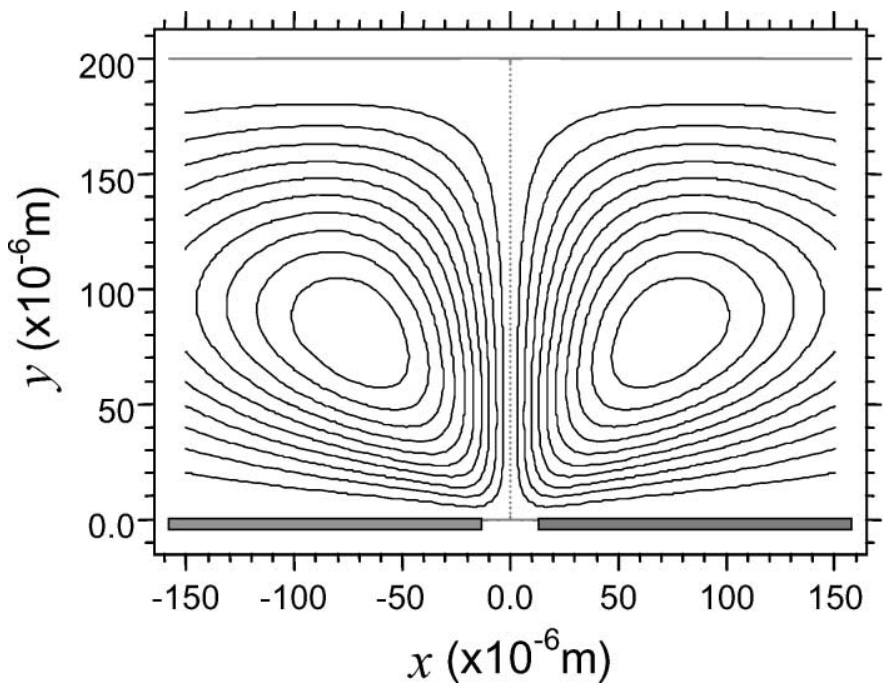

Fig. 7. The calculated streamlines for the velocity profile in Fig. 6, mirrored about the line $x=0$ to give the flow over the two electrodes. The electrodes are marked in the figure.

Again, since the equations that describe the system are linear, the velocities for other voltages and temperature increments can be found from this solution by scaling (velocity proportional to $\Delta T V^{2}$ ). 
The experimentally measured velocity in a region approximately $40 \mu \mathrm{m}$ above the centre of the gap between the electrodes was $80 \mu \mathrm{m} \mathrm{s}^{-1}$ at a potential of $10 \mathrm{~V}$ and a frequency of $1 \mathrm{MHz}$. From the numerical velocity, a vertical gradient of temperature of $0.021 \mathrm{~K} \mathrm{\mu m}^{-1}$ is required to obtain the observed velocity. This gradient of temperature implies that the temperature of the electrode is $6 \mathrm{~K}$ greater than the outside. Since the numerical model contains several major simplifications (2D space, boundary conditions), the actual temperature rise will be different but is expected to be of this order of magnitude. As pointed out in the introduction, temperature rises up to $15 \mathrm{~K}$ were measured with a thermocouple placed at the focal plane of the light. Since in the flow experiments the focal plane was somewhat higher than the level of the electrodes and the geometry and materials were different from those of the thermocouple, it is perfectly possible for the actual temperature rise to be approximately $6 \mathrm{~K}$, as predicted.

Observations were also made of the fluid flow under the condition that only one of the two electrodes was illuminated in order to demonstrate that the illumination was the factor responsible for the fluid flow [8]. This problem was also modelled numerically by expanding the problem space to include both electrodes, since in this case there is no plane of symmetry. In this case the illuminated electrode was assigned a constant temperature and the other was assumed to be transparent for the thermal problem. The streamlines are shown in Fig. 8 and agree with the experimental observations [8]. The required temperature rise for the illuminated electrode was also $\sim 6 \mathrm{~K}$ in order to obtain the experimental velocity.

The perturbative expansion that allows us to decouple the electrical and thermal equations is based on the assumption that the relative increment in conductivity (or permittivity) due to temperature for a typical distance is small. The validity of this

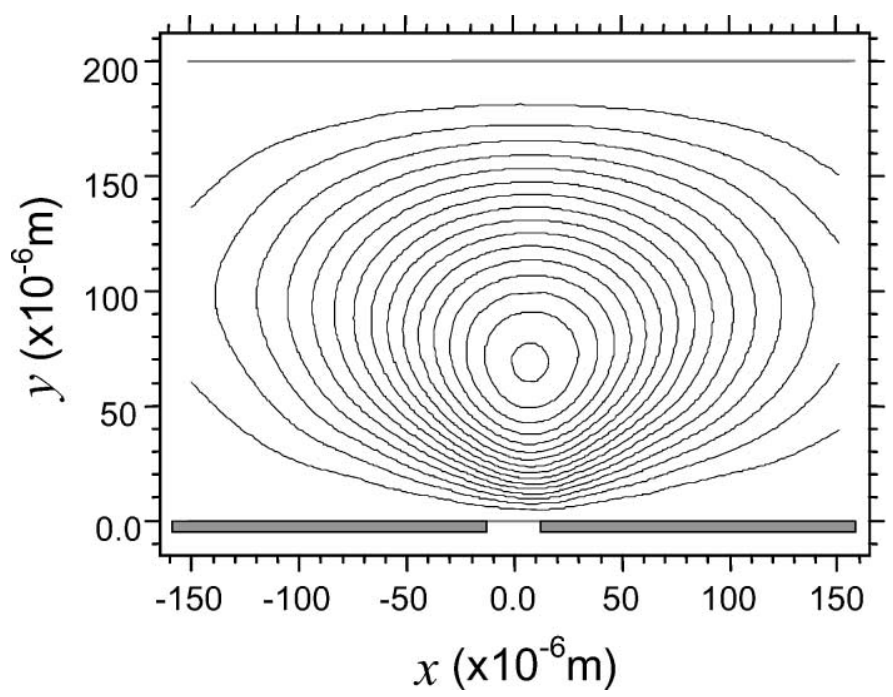

Fig. 8. The calculated streamlines from the velocity profile with only one of the two electrodes heated. The electrodes are marked in the figure. 
assumption can be confirmed as follows. The typical distance of a roll is $100 \mu \mathrm{m}$ (see Fig. 6), the maximum gradient in temperature is $0.021 \mathrm{~K} \mathrm{\mu m}^{-1}$, and the relative change in conductivity with temperature is $2 \% \mathrm{~K}^{-1}$. Then, $\Delta \sigma / \sigma=0.04$ which is much less than one, so that the simplification is justified.

\subsection{Discussion}

Fluid flow due to the two heating mechanisms is likely to occur at the same time and in competition, since the fluid flow computed for the Joule heating case moves in opposite direction to that of the high light intensities. A schematic map of the expected low-frequency behaviour depending on the applied potential and the conductivity of the fluid is shown in Fig. 9. The two solid lines indicate where an arbitrary fluid velocity $\left(10 \mu \mathrm{m} \mathrm{s}^{-1}\right.$ at $40 \mu \mathrm{m}$ above the centre of the gap) would be found, with higher fluid velocities in the shaded areas above and lower velocities below, for the two heating mechanisms. The horizontal solid line represents the flow due to light heating, determined using the experimental observations. The voltage for

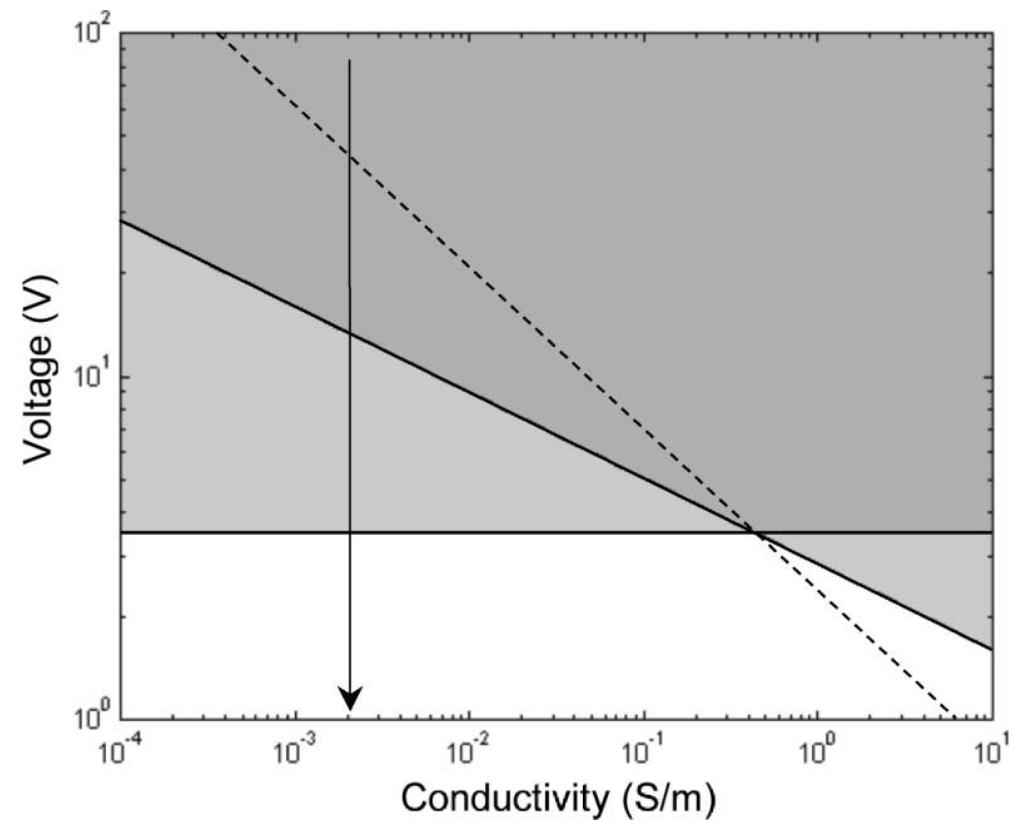

Fig. 9. A schematic diagram of the relationship between the observed light-dependent fluid flow and electrothermal fluid flow arising from Joule heating. The horizontal solid line indicates the voltage required to produce a $10 \mu \mathrm{m} \mathrm{s}^{-1}$ fluid flow for the case of light heating the electrode. This flow does not depend on the conductivity of the fluid. The sloped solid line indicates the voltage required to produce the same fluid velocity for the Joule heating case. This line has a slope of $-\frac{1}{4}$ on this $\log -\log$ plot because of the relationship $\sigma V^{4}$. The dotted line indicates the required voltage for the two flows to be of the same magnitude (in this case the actual fluid velocities vary along this line). The line with the arrow indicates the experimental conductivity for the measured values. 
this flow does not depend on the medium conductivity but depends on the intensity of the light in some manner, the line moving downwards as the intensity is increased. The second solid line represents the motion due to Joule heating in the case that the electrodes do not influence the thermal field. This line does not depend on light intensity and it is given by the relationship $\sigma V^{4}=$ Constant. The dashed line indicates when the velocities produced by the two mechanisms are equal, and it is given by the relation $\sigma V^{2}=$ Constant. As can be seen from the figure, a conductivity of $0.3 \mathrm{~S} \mathrm{~m}^{-1}$ is required for the observation of fluid motion due to Joule heating under the same illuminating conditions and voltage as the experiments. For the experimental conductivity (marked by the arrow), a voltage of $\sim 50 \mathrm{~V}$ is required to have the Joule heating fluid flow of the same order of magnitude as the illumination.

\section{Conclusions}

Electrothermally driven fluid flow has been observed on microelectrodes, arising in some manner from the illumination of the system. The electric and thermal fields for this system have been numerically calculated for the case of Joule heating and for an electrode heated by the illumination. The electrical body force due to the temperature gradients on the fluid and the resulting fluid velocity fields have also been numerically calculated. Comparisons of the numerical calculations and experimental results have demonstrated that Joule heating does not explain the observations and that the heated electrode model provides an accurate simulation of the observations if the temperature increment on the electrode is $\sim 6 \mathrm{~K}$. In this analysis we have assumed that the electrodes are heated by the light. Absorption of light by a metal is always possible, since the metal is never a perfect conductor. Also, the phenomenon known as surface plasmon resonance (excitation of electromagnetic modes along the surface of the metal) can have some influence: the transformation of incoming light into heat through dissipation of these plasmons. Experiments to investigate the absorption of light in the system and to measure the temperature rise are being performed.

\section{Acknowledgements}

The authors would like to thank the European Union for awarding a Marie Curie fellowship (contract no. BIO4-CT98-5010 (DG12-SSMI)) to N.G. Green and the Spanish Dirección General de Enseñanza Superior (research project no. BFM 20001056).

\section{Appendix A}

To examine the limitations of the Neumann boundary condition in the calculation of the electrical potential, the problem was resolved with the glass sections included, 
as for the thermal problem. In this case, Eq. (14) describes the condition at the glasswater interface and no boundary conditions are required. The variation of the potential in the fluid is now a complex problem depending on the permittivities, the conductivities and the frequency of the applied field. The error due to the assumption of a Neumann boundary condition can be examined by considering the low frequency (conductivities dominate) and high frequency (permittivities dominate) limits: the error at all frequencies will lie between the values at these two limits.

At low frequencies, the difference between the two solutions for the potential is very small. This is not unexpected as the conductivity of the suspending media is of the order of $10^{-4} \mathrm{~S} \mathrm{~m}^{-1}$ to $1 \mathrm{~S} \mathrm{~m}^{-1}$ and the conductivity of the glass is less than $10^{-8} \mathrm{~S} \mathrm{~m}^{-1}$. In this case, the validity of the Neumann boundary condition can be seen by inserting the values for conductivity and frequency into Eq. (14). In the high frequency limit, the ratio between the permittivity of water $(\sim 80)$ and glass $(\sim 2.56)$ is much less than the ratio for the conductivities. The difference between the potential calculated with Neumann boundary conditions and that calculated with a glass boundary (with water as the electrolyte) is shown in Fig. A.1. The maximum difference can be seen to be $\sim 1 \%$ of the applied voltage, which occurs at the top of the cell (furthest away from the electrodes) falling to zero at the bottom surface. The difference is negligible where the electric field is highest at the electrode edge.

We conclude that the error which arises from use of Neumann boundary conditions at the glass-water interfaces is much smaller than experimental

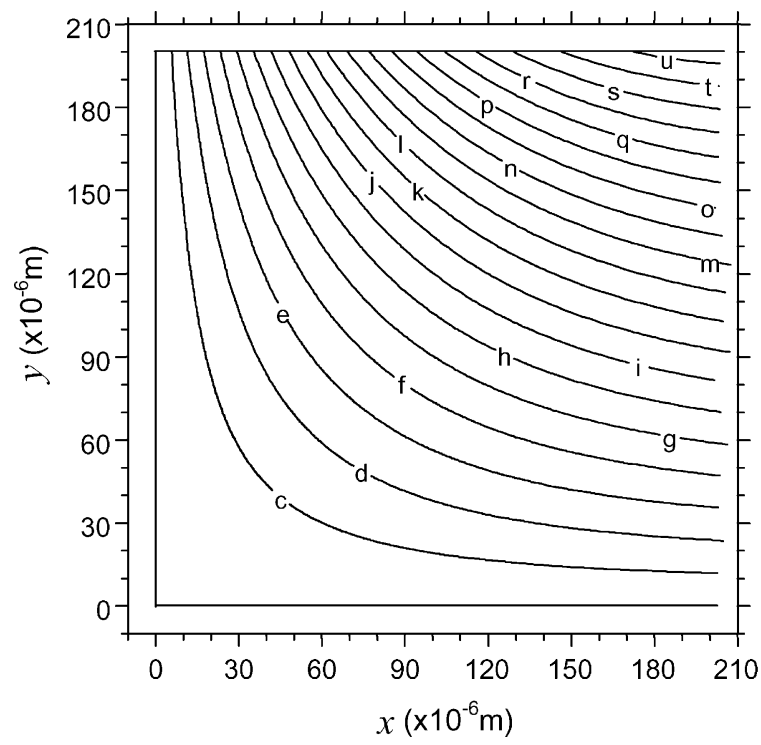

$\begin{array}{ll}\text { Scale }=10^{-3} \\ \mathrm{u}: 9.50 \\ \mathrm{t}: 9.00 \\ \mathrm{~s}: 8.50 \\ \mathrm{r}: 8.00 \\ \mathrm{q}: 7.50 \\ \mathrm{p}: 7.00 \\ \mathrm{o}: 6.50 \\ \mathrm{n}: 6.00 \\ \mathrm{~m}: 5.50 \\ \mathrm{l}: 5.00 \\ \mathrm{k}: & 4.50 \\ \mathrm{j}: & 4.00 \\ \mathrm{i}: & 3.50 \\ \mathrm{~h}: & 3.00 \\ \mathrm{~g}: & 2.50 \\ \mathrm{f}: & 2.00 \\ \mathrm{e}: & 1.50 \\ \mathrm{~d}: & 1.00 \\ \mathrm{c}: & 0.50 \\ \mathrm{~b}: & 0.00 \\ & \end{array}$

Fig. A.1. A plot of the difference between the potential calculated with Neumann boundary conditions and that calculated with a glass boundary in units of the applied voltage to the electrode. The maximum difference occurs at the top of the cell (furthest away from the electrodes) and falls to zero at the bottom surface. 
uncertainty for the data in this paper. Simplifying the solution space in this manner also means that the solution around the important region at the inter electrode gap will be improved.

\section{References}

[1] R. Pethig, Dielectrophoresis: using inhomogeneous AC electrical fields to separate and manipulate cells, Crit. Revs. Biotechnol. 16 (1996) 331-348.

[2] X.-B. Wang, Y. Huang, P.R.C. Gascoyne, F.F. Becker, Dielectrophoretic manipulation of particles, IEEE Trans. Ind. Appl. 33 (1997) 660-669.

[3] H. Morgan, M.P. Hughes, N.G. Green, Separation of submicron bioparticles by dielectrophoresis, Biophysical J. 77 (1999) 516-525.

[4] A. Ramos, H. Morgan, N.G. Green, A. Castellanos, AC electric-field induced fluid flow in microelectrodes, J. Colloid Interface Sci. 217 (1999) 420-422.

[5] N.G. Green, A. Ramos, A. Gonzalez, H. Morgan, A. Castellanos, Fluid flow induced by nonuniform AC electric fields in electrolytes on microelectrodes I: experimental measurements, Phys. Rev. E 61 (2000) 4011-4018.

[6] G. Fuhr, R. Hagedorn, T. Müller, W. Benecke, B. Wagner, Microfabricated electrohydrodynamic (EHD) pumps for liquids of higher conductivity, J. Microelectromech. Systems 1 (1992) 141-146.

[7] T. Muller, A. Gerardino, T. Schnelle, S.G. Shirley, F. Bordoni, G. DeGasperis, R. Leoni, G. Fuhr, Trapping of micrometre and sub-micrometre particles by high-frequency electric fields and hydrodynamic forces, J. Phys. D 29 (1996) 340-349.

[8] N.G. Green, A. Ramos, A. González, A. Castellanos, H. Morgan, Electric field induced fluid flow on microelectrodes: the effect of illumination, J. Phys. D 33 (2000) L13-L17.

[9] A. Ramos, H. Morgan, N.G. Green, A. Castellanos, AC electrokinetics: a review of forces in microelectrode structures, J. Phys. D 31 (1998) 2338-2353.

[10] PDE Solutions Inc. www.pdesolutions.com.

[11] J.A. Stratton, Electromagnetic Theory, McGraw-Hill, New York, 1941.

[12] J.R. Melcher, Continuum Electromechanics, MIT Press, Cambridge, USA, 1981.

[13] D.R. Lide (Ed.), CRC Handbook of Chemistry and Physics, 74th Edition, CRC Press, London, 1994.

[14] G.K. Batchelor, An Introduction to Fluid Dynamics, Cambridge University Press, Cambridge, 1967.

[15] A. Castellanos (Ed.), Electrohydrodynamics, Springer, New York, 1998 (Chapter 4).

[16] G. Backstrom, Fluid dynamics by finite element analysis, Studentlitteratur, Lund, Sweden, 1999. 